Editor's Note: These short, critical reviews of recent papers in the Journal, written exclusively by graduate students or postdoctoral fellows, are intended to summarize the important findings of the paper and provide additional insight and commentary. For more information on the format and purpose of the Journal Club, please see http://www.jneurosci.org/misc/ifa_features.shtml.

\title{
The Calcium Kinetics and Inositol Trisphosphate Receptor Properties Shape the Asymmetric Timing Window of Coincidence Detection
}

\author{
Hideaki Ogasawara \\ Computational Neuroscience Subgroup, Biological Information and Communications Technology Group, Research Department 1, National Institute of \\ Information and Communications Technology, 619-0288 Kyoto, Japan \\ Review of Sarkisov and Wang (http://www.jneurosci.org/cgi/content/full/28/1/133)
}

The cerebellum is thought to be a specialized organ for supervised learning (also known as associative learning), through which each input signal is specifically associated with a desired output. Purkinje cells provide the sole output from the cerebellar cortex, and each Purkinje cell receives two types of excitatory inputs: one from hundreds of thousands of parallel fibers and the other from a single climbing fiber. The transmission efficacy of the parallel fiber-Purkinje cell synapse is depressed when the climbing fiber and parallel fiber are repetitively and synchronously activated [long-term depression (LTD)]. This spike-timingdependent plasticity is widely accepted as the cellular correlate of cerebellar associative learning.

Parallel fibers and climbing fibers activate different signaling pathways in Purkinje cells. On the one hand, parallel fiber firing activates the mGluR1 metabotropic glutamate receptor pathway, resulting in activation of phospholipase C (PLC) and production of inositol 1,4,5trisphosphate $\left(\mathrm{IP}_{3}\right)$. However, climbing

Received Feb. 13, 2008; revised March 13, 2008; accepted March 13, 2008 I thank Kelvin So for helpful comments on this manuscript.

Correspondence should be addressed to Hideaki Ogasawara, National Institute of Information and Communications Technology, 2-2-2, Hikaridai, Seika, 619-0288 Kyoto, Japan. E-mail: h_ogasawara@nict.go.jp. DOI:10.1523/JNEUROSCI.0644-08.2008

Copyright $\odot 2008$ Society for Neuroscience $\quad$ 0270-6474/08/284293-02\$15.00/0 fiber firing depolarizes the Purkinje cell and induces $\mathrm{Ca}^{2+}$ influx through voltagegated calcium channels. Therefore, $\mathrm{IP}_{3}$ and $\mathrm{Ca}^{2+}$ represent parallel-fiber and climbing-fiber activities, respectively. By sensing sequential binding of $\mathrm{IP}_{3}$ and $\mathrm{Ca}^{2+}$, the $\mathrm{IP}_{3}$ receptor acts as a coincidence detector that associates parallel fiber inputs with climbing fiber inputs. The output signal is the release of more $\mathrm{Ca}^{2+}$ from internal stores, which leads to LTD (Wang et al., 2000; Doi et al., 2005). IP $_{3}$ receptor-mediated calcium-induced calcium release is greatest when parallel fiber stimuli precede climbing fiber stimuli by $\sim 100 \mathrm{~ms}$ (half-maximal width, $\sim 200$ $\mathrm{ms})$.

The characteristics of the $\mathrm{IP}_{3}$ receptor that shape the timing window of coincidence detection are not fully understood. To answer this question, Sarkisov and Wang (2008) explored the coincidence detector properties of the $\mathrm{IP}_{3}$ receptor in Purkinje cells by photolyzing doublecaged $\mathrm{IP}_{3}$, which is less antagonistic to the $\mathrm{IP}_{3}$ receptor and offers a higher spatial resolution of focal uncaging than its singlecaged counterpart (Sarkisov et al., 2007). Coincident presentation of $\mathrm{IP}_{3}$ and climbing fiber activation triggered calcium signals in Purkinje cells that were substantially larger than the linear sum of the signals from climbing fiber and $\mathrm{IP}_{3}$ alone [Sarkisov and Wang (2008), their Fig. 1 (http://www.jneurosci.org/cgi/content/ full/28/1/133/F1)]. Climbing fiber activation up to $100 \mathrm{~ms}$ before or up to $500 \mathrm{~ms}$ after $\mathrm{IP}_{3}$ uncaging resulted in synergistic calcium release [Sarkisov and Wang (2008), their Fig. 2 (http://www. jneurosci.org/cgi/content/full/28/1/133/ F2)]. Calcium responses were sensitive to the order of $\mathrm{IP}_{3}$ uncaging and climbing fiber activation, and the maximal signals were observed when the climbing fiber was activated 100-200 ms after $\mathrm{IP}_{3}$ uncaging; they reached $0.6-1.8 \mu \mathrm{M}$ in spines and $0.3-0.7 \mu \mathrm{M}$ in spiny dendrites [Sarkisov and Wang (2008), their Figs. $1 D$ (http://www.jneurosci.org/cgi/content/ full/28/1/133/F1), 2C,D (http://www. jneurosci.org/cgi/content/full/28/1/133/ F2)]. This optimal delay of climbing fiber inputs is consistent with their putative role in associative learning as teaching signals. Coincidence-dependent supralinearity emerged in a shorter time than enzymatic reactions take [Sarkisov and Wang (2008), their Fig. 3 (http://www. jneurosci.org/cgi/content/full/28/1/133/ F3)], which, in agreement with previous simulation studies (Doi et al., 2005; Hernjak et al., 2005), suggests that PLC is not involved in the positive feedback loop of calcium-induced calcium release.

Next, by using a calcium-store depletor and an $\mathrm{IP}_{3}$ receptor blocker, the authors demonstrated that supralinear cal- 
cium signals resulted from $\mathrm{IP}_{3}$ receptormediated calcium-induced calcium release from internal stores [Sarkisov and Wang (2008), their Fig. 4D (http://www. jneurosci.org/cgi/content/full/28/1/133/ F4)], as reported previously (Wang et al., 2000). To rule out the possibility that $\mathrm{IP}_{3}{ }^{-}$ induced calcium release activates potassium conductance and enhances climbing fiber-evoked calcium entry to the hyperpolarized dendrite, they visualized calcium signals along the dendrite. As expected, the spatial distribution of supralinear calcium responses was much more restricted than that of electrical potentials [Sarkisov and Wang (2008), their Fig. 4 (http://www.jneurosci.org/cgi/ content/full/28/1/133/F4)], indicating that potassium conductances are unlikely to be responsible for the supralinearity.

Calcium is stimulatory to the $\mathrm{IP}_{3}$ receptor at low concentrations, whereas it is inhibitory at high concentrations (Iino, 1990). Therefore, to test whether climbing fiber-evoked calcium influx had positive or negative effects on calcium release from internal stores, the authors measured calcium signals after stimulating the climbing fiber variable times and photolyzing $\mathrm{IP}_{3}$ at the end of the stimuli [Sarkisov and Wang (2008), their Fig. 5 (http://www. jneurosci.org/cgi/content/full/28/1/133/ F5)]. The more times the climbing fiber was stimulated, the greater amount of calcium was released from internal stores, indicating that the climbing fiber-evoked calcium responses in their experimental settings were within a range that stimulates the $\mathrm{IP}_{3}$ receptor.

The $\mathrm{IP}_{3}$-before-climbing-fiber timing window of several hundred milliseconds [Sarkisov and Wang (2008), their Fig. 2C,D (http://www.jneurosci.org/cgi/ content/full/28/1/133/F2)] is similar to the binding dissociation time of $\mathrm{IP}_{3}$ from its receptor, suggesting that the timing window is defined by this dissociation. $\mathrm{IP}_{3}$ degradation also might shape the timing window, although some theoretical studies have indicated that it is unlikely (Doi et al., 2005; Hernjak et al., 2005). To experimentally rule out $\mathrm{IP}_{3}$ degradation as a timing window constraint, the authors took advantage of a poorly hydrolyzable agonist of the $\mathrm{IP}_{3}$ receptor, $\mathrm{gPIP}_{2}$. gPIP uncaging at two time points revealed that the size of paired-pulse enhancement was a monotonically decreasing function of the interval, with a half-maximal width of $400 \mathrm{~ms}$, which corresponded to the bind- ing dissociation timescale [Sarkisov and Wang (2008), their Fig. 6 (http://www. jneurosci.org/cgi/content/full/28/1/133/ F6)]. This finding supports the previous hypothesis that the timing window was set by dissociation of $\mathrm{IP}_{3}$ from its receptor, not by $\mathrm{IP}_{3}$ degradation.

Collectively, these remarkable experiments indicate that the asymmetric timing window of coincidence detection in the Purkinje cell is shaped by the calcium kinetics and $\mathrm{IP}_{3}$ receptor properties; but some intriguing questions still need to be answered. First, could LTD be induced in a coincidence-dependent manner by the coincident activation protocol the authors used? If and only if so, coincidence signals explored in this study could be discussed in the context of spike-timingdependent plasticity mechanisms. The above question arises because $\mathrm{IP}_{3}$ uncaging and climbing fiber activation resulted in supralinear calcium signals [Sarkisov and Wang (2008), their Fig. 1 (http:// www.jneurosci.org/cgi/content/full/28/1/ $133 / F 1)]$ that were considerably smaller $(<2 \mu \mathrm{M})$ than those resulting from conjunctive parallel-fiber-climbing-fiber activation ( $>10 \mu \mathrm{M})$ (Wang et al., 2000). Although the authors cite another study indicating that $2 \mu \mathrm{M}$ of calcium was sufficient for LTD induction (Tanaka et al., 2007), in that study, LTD required a sustained calcium increase lasting for more than several seconds. Thus, the calcium transients that decayed within hundreds of milliseconds in Sarkisov and Wang (2008) may not have been sufficient to produce LTD.

The second remaining question concerns the order dependence of the peak supralinearity. The authors observed maximal supralinearity at $\Delta t=\sim 100 \mathrm{~ms}$ [Sarkisov and Wang (2008), their Fig. 2C,D (http://www.jneurosci.org/cgi/ content/full/28/1/133/F2)]. But if the timing window were shaped solely by the calcium kinetics and $\mathrm{IP}_{3}$ receptor- $\mathrm{IP}_{3}$ dissociation profile, the supralinearity should have been largest when $\mathrm{IP}_{3}$ uncaging and climbing fiber activation were exactly coincident, leading to a simultaneous rise of $\mathrm{IP}_{3}$ and $\mathrm{Ca}^{2+}$ concentrations and the most efficient activation of the $\mathrm{IP}_{3}$ receptor. In other words, the difference in rate between calcium decay and binding dissociation of $\mathrm{IP}_{3}$ from its receptor [Sarkisov and Wang (2008), their Fig. 7 (http:// www.jneurosci.org/cgi/content/full/28/1/ $133 / F 7)]$ only accounts for the difference in slopes on the left and right sides of each asymmetric supralinearity plot [Sarkisov and Wang (2008), their Fig. 2C,D (http:// www.jneurosci.org/cgi/content/full/28/1/ $133 / F 2)]$, leaving the deviation of its peak from the exact point of coincidence unexplained. UV-induced $\mathrm{IP}_{3}$ photolysis and climbing fiber-induced calcium influx are fast processes that take place and affect the intracellular $\mathrm{Ca}^{2+}$ concentrations within 10-20 ms [Sarkisov and Wang (2008), their Figs. 1C (http://www.jneurosci.org/ cgi/content/full/28/1/133/F1), 6 A (http:// www.jneurosci.org/cgi/content/full/28/1/ 133/F6)], and their timing does not account for the shift of the peak, either. In addition to the lack of explanatory factors for the order-dependence of the maximal supralinearity, the order-dependence itself is unclear. Fitting a Gaussian distribution to an asymmetric plot [as was done in this study Sarkisov and Wang (2008), their Fig. 2C,D (http://www.jneurosci. org/cgi/content/full/28/1/133/F2)] can lead to erroneous statistical conclusions as well as a misinterpretation of the peak location. Considering that, it might be better not to reject for now the null hypothesis that the peak of the supralinearity lies at $\Delta t=0$.

\section{References}

Doi T, Kuroda S, Michikawa T, Kawato M (2005) Inositol 1,4,5-trisphosphate-dependent $\mathrm{Ca}^{2+}$ threshold dynamics detect spike timing in cerebellar Purkinje cells. J Neurosci 25:950-961.

Hernjak N, Slepchenko BM, Fernald K, Fink CC, Fortin D, Moraru II, Watras J, Loew LM (2005) Modeling and analysis of calcium signaling events leading to long-term depression in cerebellar Purkinje cells. Biophys J 89:3790-3806.

Iino M (1990) Biphasic $\mathrm{Ca}^{2+}$ dependence of inositol 1,4,5-trisphosphate-induced $\mathrm{Ca}$ release in smooth muscle cells of the guinea pig Taenia caeci. J Gen Physiol 95:1103-1122.

Sarkisov DV, Wang SS (2008) Order-dependent coincidence detection in cerebellar Purkinje neurons at the inositol trisphosphate receptor. J Neurosci 28:133-142.

Sarkisov DV, Gelber SE, Walker JW, Wang SS (2007) Synapse specificity of calcium release probed by chemical two-photon uncaging of inositol 1,4,5-trisphosphate. J Biol Chem 282:25517-25526.

Tanaka K, Khiroug L, Santamaria F, Doi T, Ogasawara H, Ellis-Davies GC, Kawato M, Augustine GJ (2007) $\mathrm{Ca}^{2+}$ requirements for cerebellar long-term synaptic depression: role for a postsynaptic leaky integrator. Neuron 54:787-800.

Wang SS, Denk W, Häusser M (2000) Coincidence detection in single dendritic spines mediated by calcium release. Nat Neurosci 3:1266-1273. 